Portland State University

PDXScholar

\title{
Mapping of Defects in Individual Silicon Nanocrystals Using Real- Space Spectroscopy
}

\author{
Dmitry A. Kislitsyn \\ University of Oregon \\ Vancho Kocevski \\ Uppsala University \\ Jon M. Mills \\ University of Oregon \\ Sheng-Kuei Chiu \\ Portland State University, shengkuei@gmail.com \\ Christian F. Gervasi \\ University of Oregon
}

See next page for additional authors

Follow this and additional works at: https://pdxscholar.library.pdx.edu/chem_fac

Part of the Biological and Chemical Physics Commons

Let us know how access to this document benefits you.

\section{Citation Details}

Kislitsyn, D. A.; Kocevski, V.; Mills, J. M.; Chiu, S.; Gervasi, C. F.; Taber, B. N.; Rosenfield, A. E.; Eriksson, O.; Rusz, J.; Goforth, A. M.; Nazin, G. V. Mapping of Defects in Individual Silicon Nanocrystals Using RealSpace Spectroscopy. J. Phys. Chem. Lett. 2016, 7 (6), 1047-1054.

This Article is brought to you for free and open access. It has been accepted for inclusion in Chemistry Faculty Publications and Presentations by an authorized administrator of PDXScholar. Please contact us if we can make this document more accessible: pdxscholar@pdx.edu. 


\section{Authors}

Dmitry A. Kislitsyn, Vancho Kocevski, Jon M. Mills, Sheng-Kuei Chiu, Christian F. Gervasi, Benjamen N. Taber, Ariel E. Rosenfield, Olle Eriksson, Ján Rusz, Andrea Mitchell Goforth, and George V. Nazin 


\title{
Mapping of Defects in Individual Silicon Nanocrystals Using Real- Space Spectroscopy
}

\author{
Dmitry A. Kislitsyn, ${ }^{\dagger}$ Vancho Kocevski, ${ }^{\ddagger}, \|$ Jon M. Mills, ${ }^{\dagger}$ Sheng-Kuei Chiu, ${ }^{\S}$ Christian F. Gervasi, ${ }^{\dagger}$
} Benjamen N. Taber, ${ }^{\dagger}$ Ariel E. Rosenfield, ${ }^{\dagger}$ Olle Eriksson, ${ }^{\ddagger}$ Ján Rusz, $^{\ddagger}$ Andrea M. Goforth, ${ }^{\S}$ and George V. Nazin* ${ }^{\dagger}$

${ }^{\dagger}$ Department of Chemistry and Biochemistry, Materials Science Institute, Oregon Center for Optical, Molecular and Quantum Science, University of Oregon, 1253 University of Oregon, Eugene, Oregon 97403, United States

${ }^{\ddagger}$ Department of Physics and Astronomy, Uppsala University, Box 516, SE-751 20 Uppsala, Sweden

${ }^{\S}$ Department of Chemistry, Portland State University, Portland, Oregon 97201, United States

\section{Supporting Information}

ABSTRACT: The photophysical properties of silicon semiconductor nanocrystals (SiNCs) are extremely sensitive to the presence of surface chemical defects, many of which are easily produced by oxidation under ambient conditions. The diversity of chemical structures of such defects and the lack of tools capable of probing individual defects continue to impede understanding of the roles of these defects in SiNC photophysics. We use scanning tunneling spectroscopy to study the impact of surface defects on the electronic structures of hydrogen-passivated SiNCs supported on the $\mathrm{Au}(111)$ surface. Spatial maps of the local electronic density of states (LDOS) produced by our measurements allowed us to identify locally enhanced defect-induced states as well as quantum-confined states delocalized throughout the SiNC volume. We use theoretical calculations to show that the LDOS spectra associated with the observed defects are attributable to $\mathrm{Si}-\mathrm{O}-\mathrm{Si}$ bridged oxygen or $\mathrm{Si}-\mathrm{OH}$ surface defects.

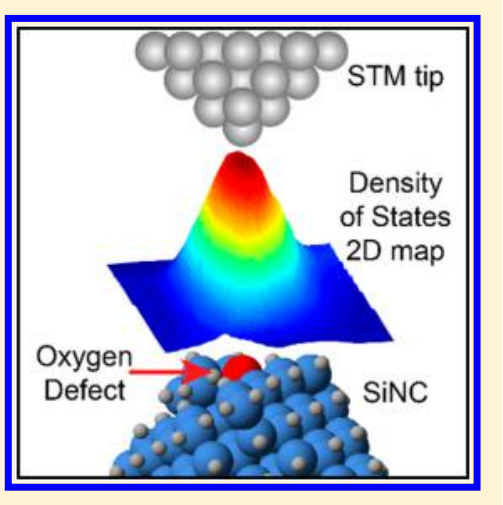

S ilicon semiconductor nanocrystals (NCs) have recently emerged as a promising alternative to metal chalcogenidebased quantum dots (QDs) in a wide range of photophysical applications including light-emitting devices ${ }^{1,2}$ and photovoltaics $^{3-6}$ as well as in biomedical imaging and tracking., Among the advantages of SiNCs are the lower toxicity of silicon and the potential for a more robust covalent passivation of SiNC surfaces. ${ }^{9,10}$ For sufficiently small SiNCs, the indirect electronic bandgap of silicon, responsible for the inefficient coupling to light in larger NCs, becomes ill-defined, ${ }^{11-13}$ which leads to a dramatic brightening of radiative transitions ${ }^{14-17}$ and thus enables optical and optoelectronic applications of SiNCs. Furthermore, multiple exciton generation in ultrasmall SiNCs has been considered as a potential route for increasing the efficiency of photovoltaic devices. ${ }^{18-20}$ While beneficial for applications, the ultrasmall size of SiNCs makes them extremely susceptible to the chemical structure of the NC surface. In particular, the tendency of SiNC surfaces to oxidize, ${ }^{21,22}$ resulting in pronounced changes in optical properties, has been a major challenge not only for applications based on SiNCs but also for the basic understanding of SiNC photophysics. The presence of oxidative defects on the SiNCs surface has been shown to produce red-shifted photoluminescence (PL), with photon energies being considerably less sensitive to the SiNC size than predicted for emission from quantum-confined states. $^{23-28}$ The exact origin of this emission remains poorly understood, which is due, in part, to the diversity of chemical defects resulting from surface oxidation of silicon ${ }^{29,30}$ and the lack of experimental techniques capable of directly identifying the chemical structures of defects responsible for the red-shifted $\mathrm{PL}$. Theoretical studies suggest that $\mathrm{Si}-\mathrm{O}-\mathrm{Si}$ bridged oxygen, ${ }^{31-33}$ as well as sufficient coverage of $\mathrm{Si}-\mathrm{OH}$ surface groups may result in red-shifted PL. ${ }^{13,34}$ Indeed, it would be natural to expect these defects to play a role in PL of SiNCs because both defects appear prominently in Fourier transform infrared spectroscopy (FTIR) of porous hydrogen-passivated SiNCs within minutes of exposure to ambient air. ${ }^{23}$ Alternatively, a red shift consistent with the experimental data may be attributed to emission from individual defects associated with surface silicon-oxygen double $(\mathrm{Si}=\mathrm{O})$ bonds, ${ }^{23,31-33,35-37}$ which have not, however, been observed in FTIR spectra of emissive SiNCs. Finally, completely oxidized SiNCs have also been shown to possess delocalized electronic states with calculated energies consistent with the experimentally observed PL. ${ }^{38}$ The plurality of theoretical models explaining the origin of red-shifted emission highlights the need for experimental studies that could directly identify the chemical nature and electronic structures of individual defects in individual SiNCs. This capability is offered by scanning tunneling spectroscopy (STS), ${ }^{39}$ which has been used to study

Received: January 26, 2016

Accepted: March 3, 2016

Published: March 3, 2016 


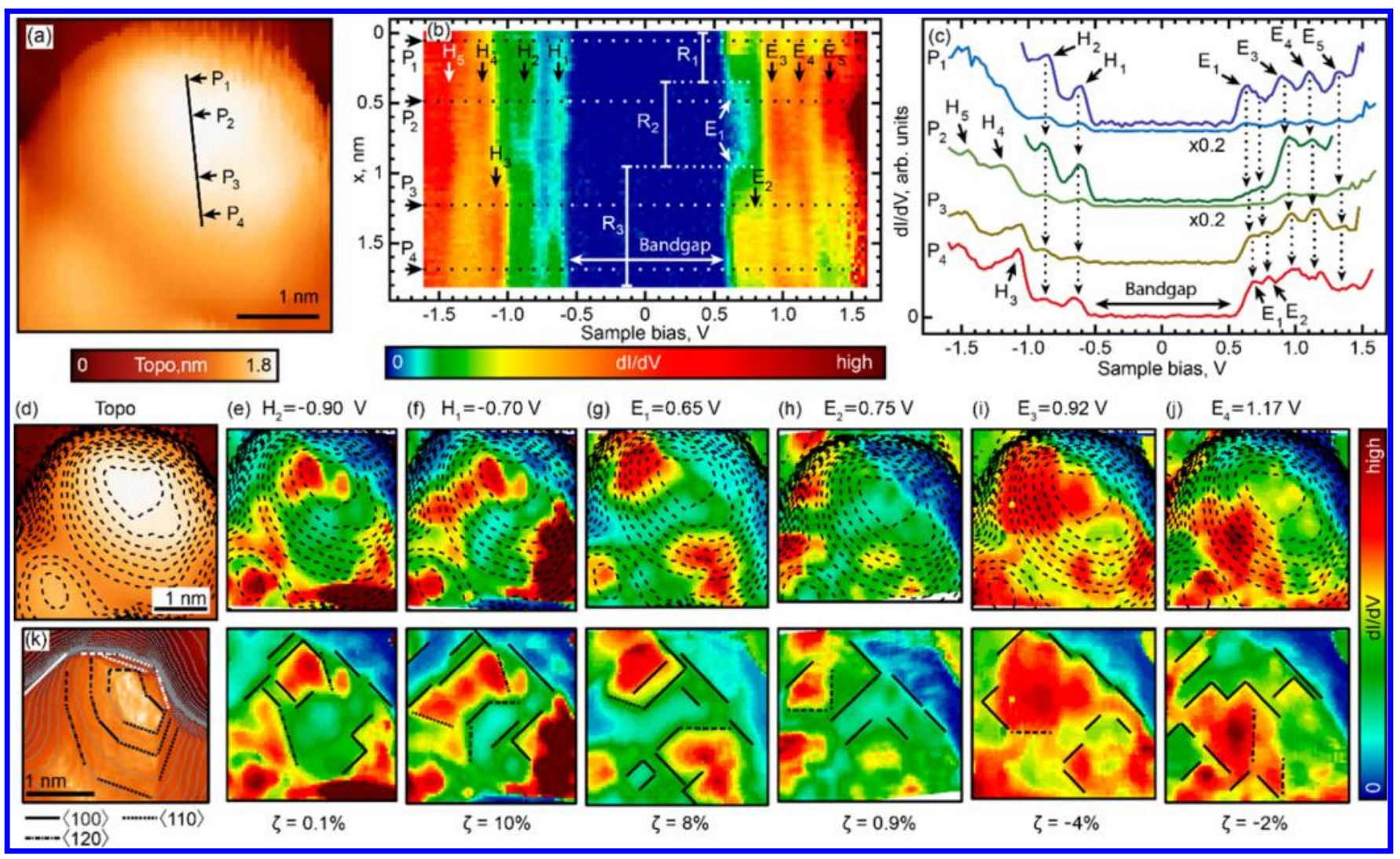

Figure 1. Spatial mapping of LDOS for $\mathrm{NC}_{1}$. (a) STM topographic image. (b) LDOS (measured as $\mathrm{d} I / \mathrm{d} V$ ) as a function of the bias voltage and position $x$ along the path shown in panel a. $R_{1}-R_{3}$ indicate regions with specific patterns of LDOS spectra. In panel $\mathrm{b}$, the individual unoccupied and occupied LDOS spectra were normalized separately, for clarity. (c) Individual LDOS spectra from panel b measured at points $\mathrm{P}_{1}$ through $\mathrm{P}_{4}$. Spectra are offset for clarity. Identical spectra for $\mathrm{P}_{1}$ and $\mathrm{P}_{2}$ scaled by $\times 0.2$ are also shown. Occupied and unoccupied states are marked " $\mathrm{H}$ " and "E", respectively, in both panels $b$ and $c$. Individual LDOS peaks are observed at slightly different voltages across the NC due to the finite locationdependent voltage drop inside the NC. (d) Topography from panel a overlaid with its contour plot. Contours correspond to vertical separation of $0.68 \AA$ (half of one $\mathrm{Si}(100)$ atomic step). (e-j) 2-D LDOS maps for voltages corresponding to the peaks indicated in panel c. Upper row shows the LDOS maps overlaid with contour lines from panel d. Lower row of LDOS maps shows Si(100) lattice directions identified in the spatial LDOS intensity distributions. Solid lines are $\langle 100\rangle$ and $\langle 010\rangle$ directions (perpendicular to each other), dashed lines are $\langle 110\rangle$ and $\langle\overline{1} 10\rangle$, dotted lines are $\langle 210\rangle$ and $\langle\overline{1} 20\rangle$, and dashed-dotted lines are $\langle 120\rangle$ and $\langle\overline{2} 10\rangle$. (Assignment of the primary directions 100 and 010 is made using the predominant orientation of linear features in the LDOS patterns.) Measure of localization, $\zeta$, is calculated for every 2-D map, as explained in the main text. (k) Calculated representation of $\mathrm{NC}_{1}$ shape (see Supporting Information for details) with its contour plot overlaid. Black and white lines on top of the contours correspond to $\mathrm{Si}(100)$ directions.

quantum confinement effects in individual $\mathrm{SiNCs}^{40}$ as well as localization of individual sub-bandgap states in $\mathrm{PbS}$ NCs. ${ }^{41,42}$

Here we report, for the first time, studies of defect-induced electronic states on the surfaces of individual hydrogenterminated SiNCs using spatially resolved STS mapping. Hydrogen-terminated SiNCs were spray-deposited onto a $\mathrm{Au}(111)$ substrate in vacuum and studied using an ultrahigh vacuum (UHV) cryogenic scanning tunneling microscope (STM) system $^{43}$ (see Methods for further experimental details). In STM images, SiNCs deposited on $\mathrm{Au}(111)$ surfaces appeared as protrusions with typical dimensions in the 2-4 nm range (Figures 1a and 3a). Using STS we recorded energydependent local density of states (LDOS) spectra of individual SiNCs by measuring the differential tunneling conductance $(\mathrm{d} I / \mathrm{d} V)$ as a function of the applied bias voltage (see Methods for details of the measurements). We focus our attention on the electronic states in the vicinity of the bandgap because these are the states that are primarily responsible for the photophysical and electron-transport properties of individual NCs and NCbased materials. In the following, we summarize our STS studies of 27 individual SiNCs.
Individual LDOS spectra measured on different SiNCs showed similar progressions of electronic states, comparable to the theoretically predicted progressions of states for hydrogenpassivated SiNCs with similar diameters (Figure S1). All SiNCs studied could be roughly divided into two classes: nominally "defect-free" SiNCs, which showed electronic states delocalized over the whole NC surface, and nominally "defect-containing" SiNCs exhibiting electronic states with LDOS strongly enhanced at specific locations. LDOS spectra of each "defectfree" SiNC contained a nearly identical set of electronic peaks $\mathrm{H}_{\mathrm{n}}$ (occupied states) and $\mathrm{E}_{\mathrm{n}}$ (unoccupied states) across the whole NC surface, as shown in Figure S2 and Figure $1 \mathrm{a}-\mathrm{c}$ for one representative $\mathrm{SiNC}$ ( $\mathrm{NC}_{1}$ in the following). The relative intensities of electronic peaks $H_{n}$ and $E_{n}$ depend on the specific spatial location, as visualized in Figure $1 \mathrm{~b}$, which shows an array of LDOS spectra recorded along the line shown in Figure 1a.

Intriguingly, the LDOS spectra (and intensities of electronic peaks) are nearly identical within specific regions on the $\mathrm{NC}_{1}$ surface (regions $R_{1}-R_{3}$ in Figure $1 b$, representative LDOS spectra for each region are shown in Figure 1c) and change on the angstrom scale at the boundaries between the regions, a 

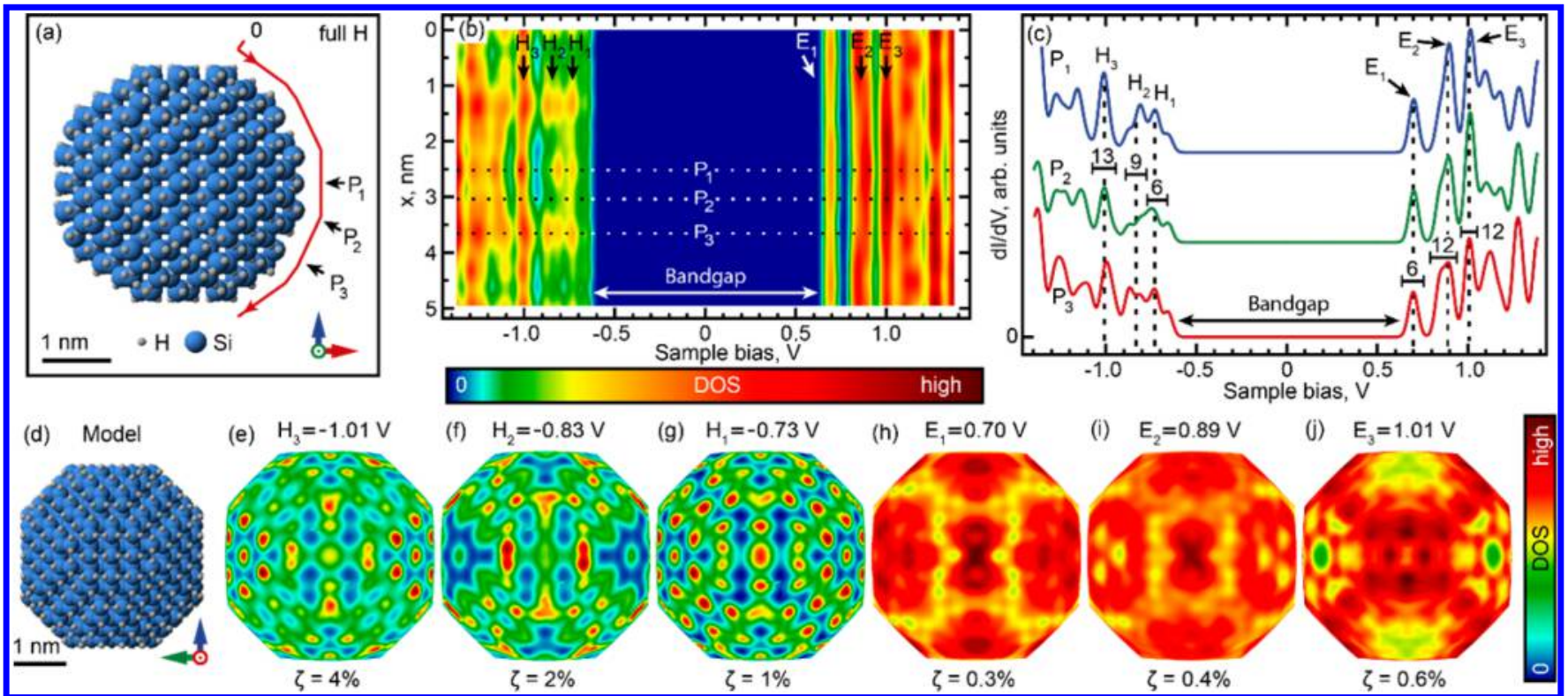

Figure 2. Theoretical LDOS for a model near-spherical (diameter $\sim 3.5 \mathrm{~nm}$ ) SiNC (with composition $\mathrm{H}_{412} \mathrm{Si}_{1087}$ ). (a) NC geometry. (b) Calculated 1-D LDOS map as a function of the bias voltage and position $\mathrm{x}$ along the path shown in panel a. (c) Individual LDOS spectra from panel $\mathrm{b}$ measured at points $\mathrm{P}_{1}$ through $\mathrm{P}_{3}$. Spectra are offset for clarity. The number of discrete states in each peak is indicated. (d) NC geometry rotated by $90^{\circ}$ around the vertical axis and exposing facets mapped in panel $b$ as well as in panels $e-j$. (e-j) 2-D maps of LDOS for selected states near the electronic bandgap. LDOS intensities were calculated on a 3-D surface equidistantly offset from the NC surface by $3 \AA$, similarly to the path shown in panel a. To more closely reproduce experimental conditions, all spectra were normalized to give the same total current at $1.35 \mathrm{~V}$ (see Methods for details). Measure of localization, $\zeta$, is calculated for every 2-D map, as explained in the main text.

behavior that was also observed for other "defect-free" SiNCs (data not shown). To further explore the nature of peaks $\mathrm{H}_{n}$ and $\mathrm{E}_{\mathrm{n}}$, we recorded 2-D spatial LDOS maps showing the distribution of each electronic state across the $\mathrm{NC}_{1}$ surface (Figure $1 \mathrm{e}-\mathrm{j}$ ). To ensure that we could identify all of the (possibly localized) electronic states in SiNCs, we recorded LDOS spectra on a 2-D grid of points covering the spatial range corresponding to Figure $1 \mathrm{~d}$. The 2-D maps in Figure $1 \mathrm{e}-\mathrm{j}$ were then generated by taking subsections of this LDOS data set corresponding to specific energies (voltages). Thus obtained 2$\mathrm{D}$ LDOS maps show that the main states of $\mathrm{NC}_{1}$ are completely delocalized (showing nonzero LDOS) on the observable part of the $\mathrm{NC}_{1}$ surface, even though 2-D-regions of varied intensities (similar to $1 \mathrm{D}$-regions $\mathrm{R}_{1}-\mathrm{R}_{3}$ in Figure $1 \mathrm{~b}$ ) are present. Strikingly, the boundaries of these regions are aligned along specific directions attributable to a $\{100\}$ crystallographic facet (bottom Figure 1e-j; see caption for details), an observation that was common for the majority of SiNCs studied. (In addition to SiNCs showing crystallographic directions attributable to $\{100\}$ facets, SiNCs showing directions consistent with $\{111\}$ facets were also observed.)

A crystallographic origin of directional order in the 2-D LDOS maps is further supported by our analysis of the STM images of the corresponding SiNCs. In this analysis, we attempted to deconvolute the effects of STM tip-shape and finite tip-NC distance $(\sim 0.8 \mathrm{~nm})$, which both lead to broadening and rounding of the apparent NC shapes observed in the STM images (see Figure S3 for details). Thus deconvoluted images show sharply defined facets (see Figure $1 \mathrm{k}$ for $\mathrm{NC}_{1}$ ) with directions identical to those found in the LDOS maps (these directions are also present in as-recorded STM images; see Figure 1d), which strongly suggests that the observed directional order in both 2-D maps and recalculated
STM images originates from the crystallographic structures of the SiNCs.

The correlation with the crystallographic directions and the delocalized nature of electronic states in "defect-free" SiNCs together suggest that these states correspond to quantumconfined states delocalized throughout the bulk of $\mathrm{NC}_{1}$ rather than defect states. (The latter, in contrast, can be expected to show LDOS enhanced at the defect locations.) To test this hypothesis, we carried out density functional theory (DFT) calculations (see Methods for details) for a set of model NCs of different sizes. For the simulations, we chose approximately spherical NC shapes (Figure 2a), as expected for SiNCs with sizes below $\sim 6 \mathrm{~nm} .{ }^{44,45}$

In agreement with the measured 2-D LDOS maps, our calculations for defect-free SiNCs (see Figure 2 for a SiNC with a size of $\sim 3.5 \mathrm{~nm}$, similar to that of $\mathrm{NC}_{1}$ ) reproduce the progressions of electronic peaks $\mathrm{H}_{\mathrm{n}}$ and $\mathrm{E}_{\mathrm{n}}$ observed in LDOS of studied SiNCs. We note that each peak in Figure $2 b, c$ is composed of several closely spaced distinct electronic states formed from states associated with different electronic valleys in the Brillouin zone of bulk $\mathrm{Si}^{46}$ These states are not completely resolved in our measurements, even though their presence is suggested by the fine structure of LDOS spectra (Figure S2c,d). Finer spectral structure could not be observed due to the intrinsic broadening of STS spectra associated with coupling of tunneling electrons to vibrational excitations, ${ }^{47}$ which exceeds the intervalley splitting for simulated SiNCs $(\sim 50 \mathrm{meV})$.

Significantly, our calculations reproduce the existence of distinct surface regions with specific LDOS spectra (Figure $2 \mathrm{~b}, \mathrm{c})$, and the presence of directional order in the spatial LDOS distributions (Figures $2 \mathrm{e}-\mathrm{j}$ ), even though the precise spatial patterns for experimental and theoretical 2-D maps are not identical. The differences in the two data sets are attributable to the fact that the precise shape of $\mathrm{NC}_{1}$, which has a profound 


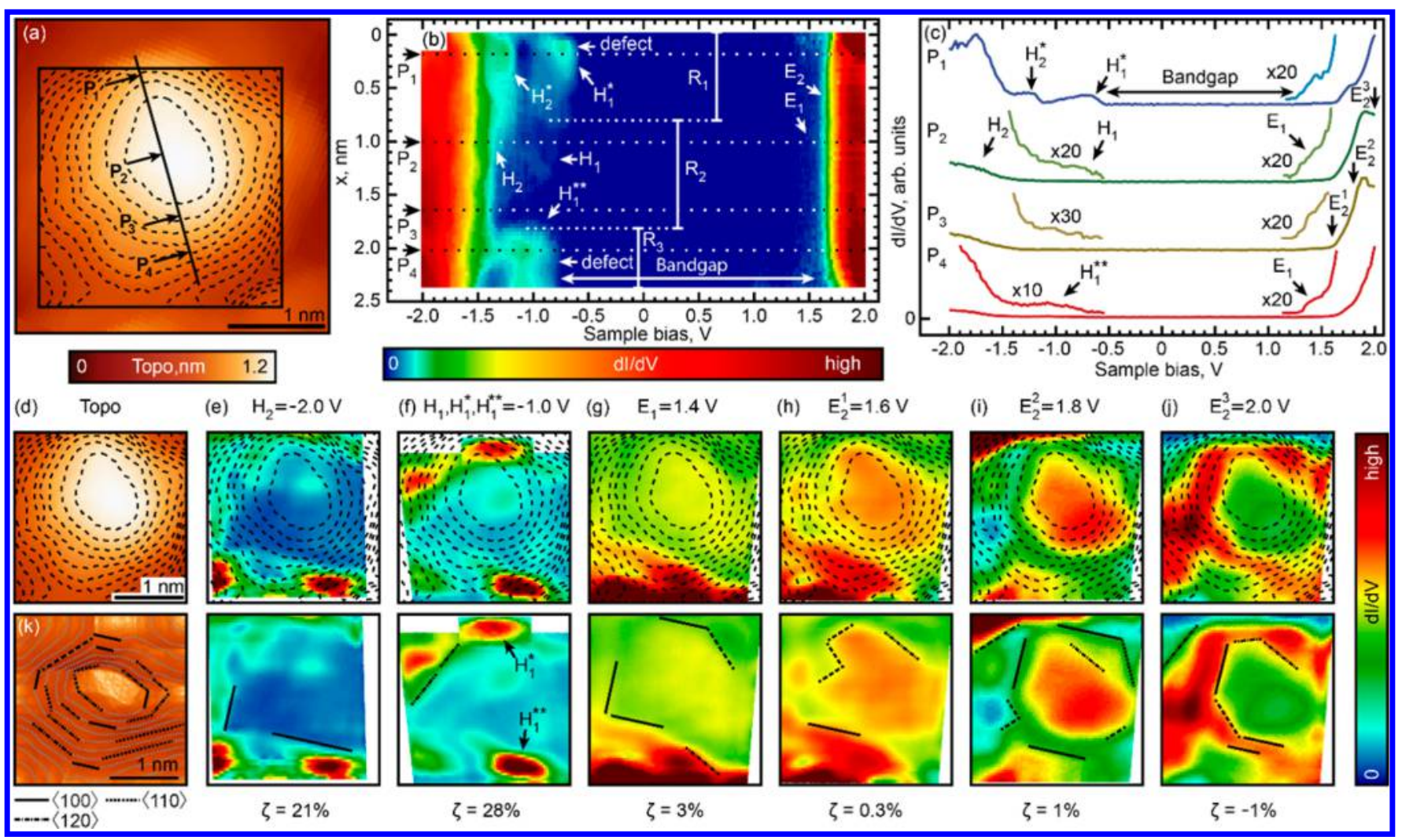

Figure 3. Spatial mapping of LDOS for $\mathrm{NC}_{2}$. Data arrangement and markings are the same as in Figure 1 . In panel $b$, the individual unoccupied and occupied LDOS spectra were normalized separately, for clarity. Peak $E_{2}$ is measured at three voltages $E_{2}^{n}$.

impact on the electronic LDOS spatial distribution, is not known (because a large part of the $\mathrm{NC}_{1}$ surface is not observable in STM imaging) and thus could not be modeled directly. The close similarity between the theoretical and experimental results in Figures 1 and 2 reinforces the interpretation of states in Figure 1 as those of quantumconfined nature, and delocalized in the interior of the $\mathrm{NC}$, analogously to the calculated 3-D LDOS distributions shown in Figures S4 and S5.

In addition to "defect-free" SiNCs with delocalized electronic states, we have also observed SiNCs (6 out of 27 SiNCs studied in our experiments) showing localized areas with strongly enhanced LDOS. An example of this behavior is shown in Figure 3 and Figure $\mathrm{S} 6$ for a representative $\mathrm{SiNC}\left(\mathrm{NC}_{2}\right.$ in the following). For example, a 1-D LDOS map (Figure 3b) of $\mathrm{NC}_{2}$ shows peaks $\mathrm{E}_{2}$ and $\mathrm{H}_{2}$ delocalized over the entire spatial range of the scan, while peaks $\mathrm{H}_{1} *$ and $\mathrm{H}_{1} * *$ (corresponding to a smaller bandgap) are primarily concentrated in regions $\mathrm{R}_{1}$ and $R_{3}$, respectively. Closer inspection reveals peaks $E_{1}$ and $H_{1}$ with lower intensities everywhere along the mapping path (Figure $3 c$ ). The 2-D LDOS maps of these electronic states (Figure $3 e-j)$ give a more complete picture: States $E_{n}$ and $\mathrm{H}_{n}$ of $\mathrm{NC}_{2}$ appear over the entire observable part of $\mathrm{NC}_{2}$, showing 2-D regions of varied intensities. Similarly to the directional order in 2-D LDOS maps of $\mathrm{NC}_{1}$, the boundaries of some of these regions are aligned along the main crystallographic directions of $\mathrm{NC}_{2}$ (bottom Figure $3 \mathrm{e}-\mathrm{j}$ ). Importantly, spatial distributions of several LDOS features appear very different from those of $\mathrm{NC}_{1}$. In particular, pronounced spatially confined maxima $\mathrm{H}_{1} *, \mathrm{H}_{1} * *$, and $\mathrm{H}_{2}{ }^{*}$ are observed in Figure 3e,f, with the spatial LDOS concentration in the corresponding maps being significantly higher than that in the other maps for $\mathrm{NC}_{1}$ and $\mathrm{NC}_{2}$. To analyze this localization quantitatively, we implemented a comparison of average and median values for a particular LDOS map, which is sensitive to the presence of sharp intense peaks in DOS maps. We constructed a measure of localization of the form: $\zeta=\frac{\overline{D O S}-\overline{D O S}}{\overline{D O S}+\overline{D O S}} \times 100 \%$ (where $\overline{D O S}$ and $\widetilde{D O S}$ are the average and median values, respectively), which for more spatially concentrated peaks $\mathrm{H}_{1}{ }^{*}, \mathrm{H}_{1}{ }^{*}$, and $\mathrm{H}_{2}{ }^{*}$ gives values $\zeta$ $\geq 20 \%$ (bottom Figure 3e,f), while the rest of the maps shows $|\zeta| \leq 10 \%$ (as listed in the respective Figures), which we consider to be representative of "delocalized" states. The spatial concentration of peaks $\mathrm{H}_{1} *, \mathrm{H}_{1} * *$, and $\mathrm{H}_{2} *$ suggest that they may correspond to defect-related states. (Because $\mathrm{H}_{1} *$ and $\mathrm{H}_{1}^{* *}$ are spatially well-separated, they likely correspond to two different defects.)

To investigate the nature of observed locally enhanced peaks $\mathrm{H}_{1}{ }^{*}$ and $\mathrm{H}_{1}{ }^{* *}$, we carried out DFT calculations of the electronic structures of SiNCs containing individual chemical defects. ${ }^{33}$ Because a substantial fraction $(\sim 22 \%)$ of studied SiNCs showed defects of the type shown in Figure 3; these are likely to be of the same chemical nature and must be very common for the hydrogen-passivated SiNCs used in our study. Surface oxidation is particularly common for such SiNCs and is known to produce a variety of oxygen-containing bonds on the SiNC surfaces, often leading to the formation of intragap states. $^{23,31,35}$ We thus restrict our consideration to elementary defects caused by oxidation, which include three main classes: $\mathrm{Si}-\mathrm{O}-\mathrm{Si}$ bridged oxygen, $\mathrm{Si}-\mathrm{OH}$ groups, and $\mathrm{Si}=\mathrm{O}$ bonds. For SiNCs studied in our experiments, the most likely candidate is the $\mathrm{Si}-\mathrm{O}-\mathrm{Si}$ bridged oxygen defect, which is the only type of defect identifiable in the FTIR spectra of assynthesized hydrogen-passivated SiNCs used in our study. ${ }^{48,49}$ 

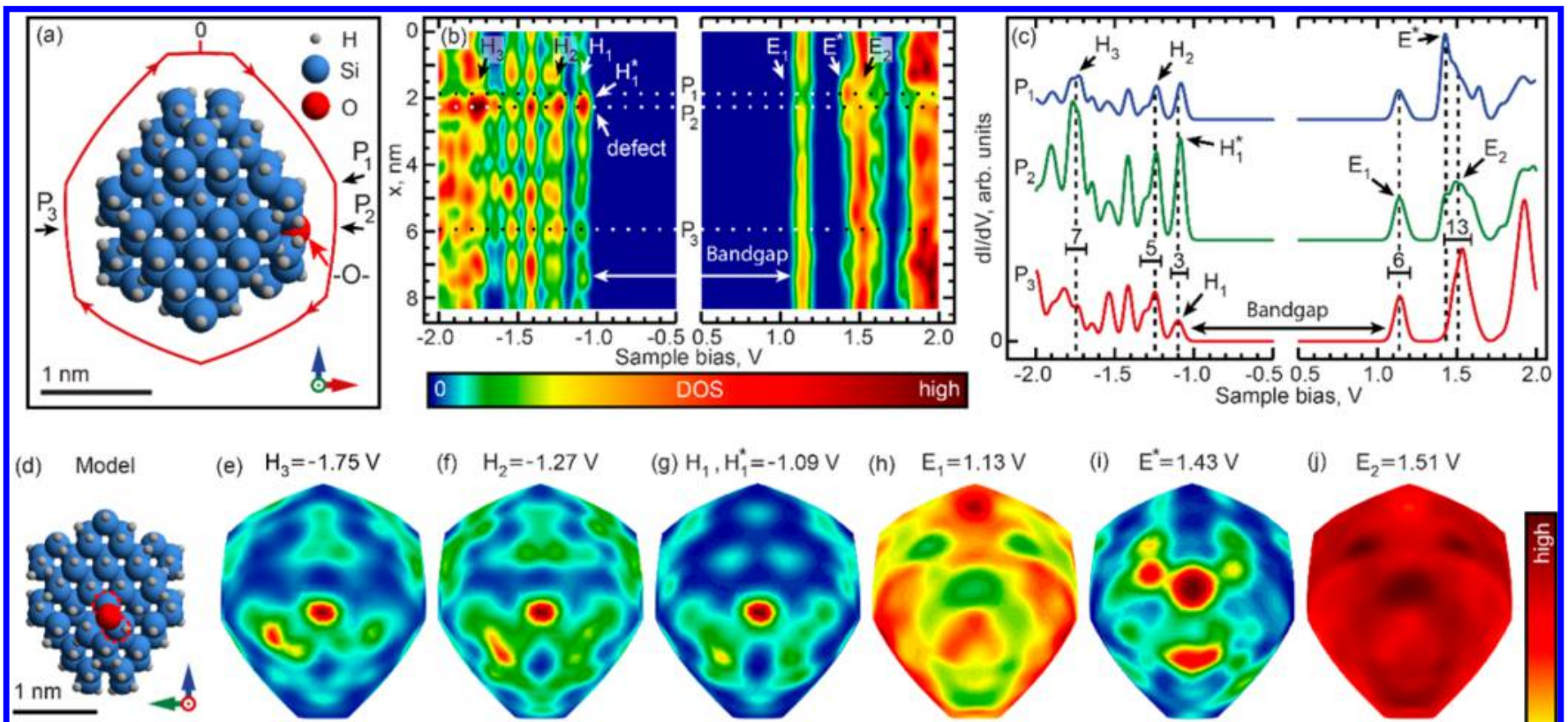

(i) $E^{*}=1.43 \mathrm{~V}$

(j) $E_{2}=1.51 \mathrm{~V}$
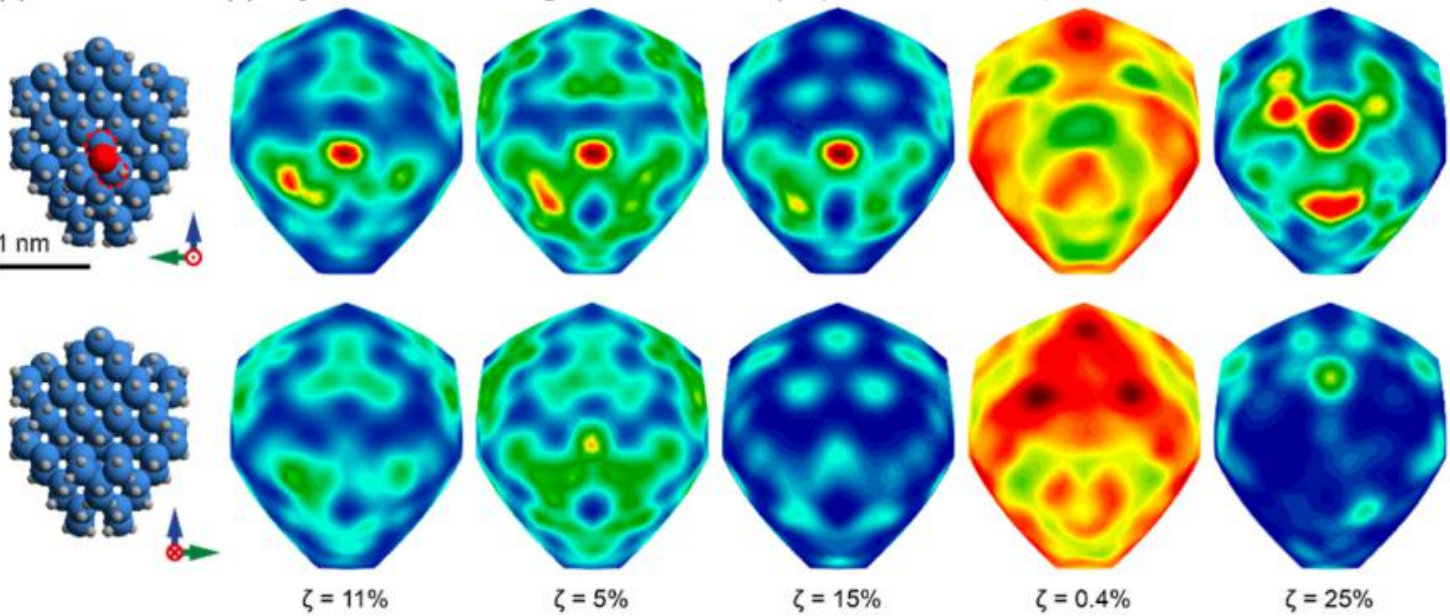

Figure 4. Theoretical LDOS for a model near-spherical (diameter $\sim 2 \mathrm{~nm}$ ) SiNC with a bridged oxygen ( $\mathrm{Si}-\mathrm{O}-\mathrm{Si}$ ) impurity and composition $\mathrm{H}_{114} \mathrm{Si}_{175} \mathrm{O}$. Data arrangement and markings are the same as in Figure 2. In panel c, $\mathrm{P}_{1}$ corresponds to the maximum of orbital $\mathrm{E}^{*} \mathrm{LDOS}, \mathrm{P}_{2}$ corresponds to the defect location, and $\mathrm{P}_{3}$ is positioned on the opposite side of $\mathrm{NC}$ with respect to $\mathrm{P}_{2}$. The top and bottom rows in panels $\mathrm{d}-\mathrm{j}$ show two opposite sides of the NC, respectively. To more closely reproduce experimental conditions, all spectra were normalized to give the same total current at $1.7 \mathrm{~V}$.

Another possible candidate are the $\mathrm{Si}-\mathrm{OH}$ surface groups, which have been identified in FTIR spectra of SiNCs synthesized using a similar technique; ${ }^{30}$ however, the presence of these defects in our SiNCs may be somewhat less likely due to their instability under vacuum conditions. ${ }^{50}$ Surface $\mathrm{Si}=\mathrm{O}$ defects have been implicated as a possible source of the redshifted defect-induced $\mathrm{PL}^{23,31,35}$ and have been observed on the SiNC surfaces under vacuum conditions. ${ }^{50}$

Theoretical simulations of 1-D LDOS maps for the $\mathrm{Si}=\mathrm{O}$ defect on the surface of a model SiNC with a size of $\sim 2 \mathrm{~nm}$, similar to that of $\mathrm{NC}_{2}$, show two peaks enhanced at the defect location: the highest-occupied peak $\mathrm{H}_{1}{ }^{*}$ and the lowestunoccupied peak $\mathrm{E}_{1}{ }^{*}$ (Figure $\mathrm{S} 7 \mathrm{~b}$; see also Figure $\mathrm{S} 7 \mathrm{~g}, \mathrm{~h}$ ). Comparison of a 1-D LDOS map of this NC (Figure S7b) to those of its defect-free variant (Figure S8b) shows that the bandgap of the defective $\mathrm{NC}$ is reduced at the defect location by $\sim 300 \mathrm{meV}$; however, the energy differences between the delocalized peaks $\mathrm{H}_{1}$ and $\mathrm{E}_{1}$ are nearly identical for both NCs, suggesting that the defect-induced peaks $\mathrm{H}_{1}{ }^{*}$ and $\mathrm{E}_{1} *$ effectively correspond to trap states within the defect-free bandgap. In contrast, bandgaps obtained from theoretical LDOS of both $\mathrm{Si}-\mathrm{O}-\mathrm{Si}$ and $\mathrm{Si}-\mathrm{OH}$ defects (Figure $4 \mathrm{~b}$ and Figure S9b, respectively) are quite similar to that of the defectfree SiNC (Figure S8b), consistent with previous theoretical results. ${ }^{23,31,33,33}$ The impact of these defects is primarily in the redistribution of the SiNC LDOS, resulting in enhanced LDOS at the location of the corresponding defect (compare peaks $\mathrm{H}_{1}$ with maxima $\mathrm{H}_{1}{ }^{*}$ in Figure $4 \mathrm{e}-\mathrm{g}$ and Figures $\mathrm{S} 9 \mathrm{e}-\mathrm{g}$ and $\mathrm{S} 8 \mathrm{e}-$ g). The varied impacts of the different types of defects on the SiNC LDOS are directly related to the specific spatial distributions of the corresponding electronic states (Figures S10-S15). Indeed, in the case of the $\mathrm{Si}=\mathrm{O}$ defect, the bandgap-forming states $\mathrm{H}_{1} *$ and $\mathrm{E}_{1} *$ are tightly localized (in three dimensions) within the immediate vicinity of the defect (Figure S11), while the bandgap-forming states for the Si-O$\mathrm{Si}$ and $\mathrm{Si}-\mathrm{OH}$ defects are delocalized within the NC interiors, with only a small fraction of the total DOS found in the defect vicinity (Figures S12-S15), reminiscent of the LDOS distributions for the defect-free NC (Figures S16 and S17).

Analysis of the specific spatial LDOS distributions expected for the different oxidative defects previously discussed (Figure 4 and Figures S7 and S9) allows us to shed light into the nature of the defect-induced states shown in Figure 3. In particular, the presence of the locally enhanced peak $\mathrm{E}_{1} *$ in the theoretical LDOS is incompatible with the experimental LDOS for the lowest unoccupied state $\mathrm{E}_{1}$ of $\mathrm{NC}_{2}$ (Figure $3 \mathrm{~g}$ ), where only a small local maximum, instead of a strongly locally enhanced peak, is observed at the defect location corresponding to peak $\mathrm{H}_{1}$ ** in Figure $3 \mathrm{f}$, and no maximum is found at the location of peak $\mathrm{H}_{1} *$ in Figure 3f. The same conclusion is suggested by the fact that in Figure $3 \mathrm{~g}$ the localization parameter $\zeta$ is dramatically smaller than those found for Figure S7h or Figure 3 f ( 3 versus 32 and $28 \%$, respectively). The absence of strong local DOS maxima in Figure $3 \mathrm{~g}$ thus suggests that the $\mathrm{Si}=\mathrm{O}$ 
defects are unlikely to be responsible for the locally enhanced states observed in Figure 3. In contrast, we find that for the Si$\mathrm{O}-\mathrm{Si}$ and $\mathrm{Si}-\mathrm{OH}$ defects, the agreement between the experimental and theoretical DOS is significantly better: The redistributions of the SiNC LDOS, resulting in enhanced occupied LDOS at the location of the corresponding defect (peaks $\mathrm{H}_{1}$ with local maxima $\mathrm{H}_{1}{ }^{*}$ in Figure 4 and Figure S9), are analogous to that found in the experimental LDOS for $\mathrm{NC}_{2}$ (peak $\mathrm{H}_{1}$ with maxima $\mathrm{H}_{1} *$ and $\mathrm{H}_{1} * *$ in Figure 3 ). Furthermore, in both experimental and theoretical data, the highest occupied states are not completely localized at the defect locations but persist over the whole NC surface (compare, for example, Figures $3 \mathrm{f}$ and $4 \mathrm{~g}$ and Figure S9g). The unoccupied states in the theoretical LDOS remain relatively uniform across the NC surface, which is also consistent with the unoccupied states in the experimental LDOS of $\mathrm{NC}_{2}$ (compare, for example, Figures $3 \mathrm{~g}-\mathrm{j}$ and $4 \mathrm{~h}, \mathrm{j}$ and Figure S9h,i). The unoccupied states in the theoretical LDOS are not significantly modified as compared with a completely hydrogen-passivated SiNC (Figures S8, S16, and $\mathrm{S} 17$ ), except for some band splitting in $\mathrm{E}_{2}$ (Figure $4 \mathrm{~b}$ and Figure $\mathrm{S} 9 \mathrm{~b}$ ), which is more noticeable for $\mathrm{Si}-\mathrm{O}-\mathrm{Si}$, a consequence of a stronger geometric perturbation resulting from this type of defect. ${ }^{31}$ In addition, in the case of $\mathrm{Si}-\mathrm{O}-\mathrm{Si}$, this splitting produces an orbital $\mathrm{E}^{*}$ concentrated around the defect (Figure 4i). This orbital, however, is not resolved in the experimental LDOS, which is likely due to the electronvibrational band broadening effect previously discussed. Significantly, the trends found for the localization parameter $\zeta$ in Figure 4 and Figure S9 are similar to those found in Figure 3. (We note that we do not expect the theoretical and experimental values of $\zeta$ to be in precise quantitative agreement because the corresponding NC structures and surfaces used for calculation of $\zeta$ are different.) The close qualitative similarities between the theoretical and experimental LDOS suggest that the defects of the type corresponding to peaks $\mathrm{H}_{1} *$ and $\mathrm{H}_{1} * *$ in Figure $3 \mathrm{~b}$ may be associated with either $\mathrm{Si}-\mathrm{OH}$ or $\mathrm{Si}-\mathrm{O}-\mathrm{Si}$ defects. (The latter is more likely, as previously mentioned.)

Defects of the type found for $\mathrm{NC}_{2}$ were observed only on SiNCs with relatively small diameters (less than $\sim 2.5 \mathrm{~nm}$ ). In contrast, nominally "defect-free" SiNCs (with electronic states delocalized) similarly to those found for $\mathrm{NC}_{1}$ were typically larger. The lack of clearly identifiable defects on larger SiNCs does not mean that these SiNCs were entirely defect-free: Our calculations show that the impact of elementary defects on the spatial LDOS patterns of such SiNCs is minimal and nearly nonexistent (for states close to the electronic bandgap) for sufficiently large SiNCs (Figure S18), which suggests that the LDOS in Figure 1 may be spatially modulated by defects, even though the presence of such defects could not be unequivocally established from our measurements.

Spatial mapping of the LDOS of individual SiNCs and theoretical calculations reported in this work show that oxidative defects spatially modulate the LDOS on SiNC surfaces producing LDOS patterns of varied degrees of inhomogeneity that depend on the NC size. For sufficiently small SiNCs, locally enhanced LDOS attributable to either Si$\mathrm{OH}$ or $\mathrm{Si}-\mathrm{O}-\mathrm{Si}$ defects are observed in LDOS mapping and reproduced in theoretical calculations. In contrast, spatial LDOS distributions (and, consequently, the photophysical properties) of larger SiNCs are relatively insensitive to the presence of such defects. The present work was carried out using an ultrastable closed-cycle cryogenic STM system, ${ }^{43}$ which uniquely enables detailed STS mapping of statistically significant quantities of individual NCs. We anticipate that further STS studies of NCs using our approach will contribute to building a more complete picture of the diverse types of defect states, in particular, dangling bond defects, which are known to significantly affect the photophysical processes in SiNCs. ${ }^{51,52}$

\section{METHODS}

Experiments were carried out in a home-built ultrahigh vacuum (UHV) cryogenic STM system. ${ }^{43} \mathrm{~A} \mathrm{Au}(111) /$ mica substrate was prepared in situ using multiple neon sputter/anneal cycles. Hydrogen-passivated SiNCs were fabricated via thermal disproportionation in a polymeric sol-gel hydrosilicate precursor and subsequently liberated from the resulting oxide host matrix via a wet chemical etch (i.e., with $\mathrm{HF} / \mathrm{EtOH} / \mathrm{H} 2 \mathrm{O}$ ) using a protocol previously described. ${ }^{48}$ (For further details see the Supporting Information.) The SiNCs were suspended in pentane, and deposited onto the $\mathrm{Au}(111)$ surface under highvacuum conditions using a solenoid pulse-valve. The deposition parameters were chosen so as to obtain monolayer NC coverage. The $\mathrm{Au}(111)$ substrate with deposited SiNCs was then degassed overnight in $\mathrm{UHV}$ at $\sim 50{ }^{\circ} \mathrm{C}$ to remove any residual pentane from the sample surface. All STM topographies and STS measurements were obtained at a temperature of $\sim 20 \mathrm{~K}$ using silver tips prepared by electrochemical etching and subsequent sputtering in UHV. All STS spectra were recorded using the lock-in technique at $570 \mathrm{~Hz}$ and a bias modulations varying from 10 (individual spectra and 1-D spatial scans) to $50 \mathrm{mV}$ (2-D LDOS maps). 2-D LDOS mapping requires tip-sample spatial registry throughout the mapping process (typically, several hours). This capability is enabled by the unique spatial stability (better than $\sim 0.2 \AA / \mathrm{h}$ ) of our STM system. ${ }^{43}$

Theoretical calculations were performed using DFT, as implemented in the pseudopotential package SIESTA, ${ }^{53,54}$ employing local density approximation (LDA) exchange and Ceperlay-Alder parametrization of the potential, as previously described. ${ }^{33}$ Numerical atomic orbitals were used as a basis set: "single zeta with polarization" orbitals for Si and O and "double zeta" for H. Further details of the basis sets for different elements are given in ref 12 . For hydrogenated SiNCs smaller than $4 \mathrm{~nm}$ in diameter, the described approach provides a good approximation of electronic bandgaps. ${ }^{12}$

To simulate the experimental data, we calculated LDOS as the probability density sum $\operatorname{LDOS}(x, y, z, V)=\sum_{j}\left|\Psi_{j}(x, y, z)\right|^{2}$ $\delta\left(e V-E_{j}\right)$ of individual Kohn-Sham orbitals $\Psi_{j}(x, y, z)$ obtained from DFT. To account for finite spatial and spectral resolution of the experimental data, the contributions of individual orbitals to the total LDOS were Gaussian-broadened by $50 \mathrm{meV}$ in energy and by $0.2 \mathrm{~nm}$ in space. To more closely reproduce experimental conditions where the integral of each $\mathrm{d} I / \mathrm{d} V$ spectrum is equal to the set point current, we normalized calculated LDOS spectra for each specific spatial location such as to yield integrals $I(x, y, z)$ equal to the set point value $I_{\mathrm{sp}}$

$$
I_{\mathrm{sp}}=I(x, y, z)=\int_{0}^{V_{\mathrm{B}}} \operatorname{LDOS}(x, y, z, V) \mathrm{d} V
$$

Here $I(x, y, z)$ represents the calculated tunneling current in each spatial location and $V_{\mathrm{B}}$ is the set point bias voltage (noted in the corresponding Figure captions). 


\section{ASSOCIATED CONTENT}

\section{S Supporting Information}

The Supporting Information is available free of charge on the ACS Publications website at DOI: 10.1021/acs.jpclett.6b00176.

Additional STM images and STS spectra of SiNCs. Calculations of LDOS for model SiNCs. Synthesis and characterization of SiNCs. (PDF)

\section{AUTHOR INFORMATION}

\section{Corresponding Author}

*E-mail: gnazin@uoregon.edu.

\section{Present Address}

"V.K.: Department of Materials Science \& Engineering, Northwestern University, Evanston, Illinois 60208, United States

\section{Notes}

The authors declare no competing financial interest.

\section{ACKNOWLEDGMENTS}

The STM instrument used in this work was constructed with support from the National Science Foundation under Grant DMR-0960211. B.N.T. and G.V.N. gratefully acknowledge support from the Center for Sustainable Materials Chemistry through the NSF CCI Grant CHE-1102637. D.A.K., J.M.M., and C.F.G. acknowledge support from NSF Grant CHE1454036. A.M.G acknowledges the Burroughs Wellcome Fund (Award Number 1007294.01) for financial support. O.E. acknowledges support from the Swedish Research Council and STANDUPP. J.R. acknowledges support from the Swedish Research Council and the Göran Gustafsson's Foundation. V.K. acknowledges support from the EU's seventh Framework Programme SNAPSUN, the Swedish National Infrastructure for Computing (SNIC), and eSSENCE. We thank Jeffery Cina for his comments on the manuscript.

\section{REFERENCES}

(1) Xin, Y.; Nishio, K.; Saitow, K.-i. White-Blue Electroluminescence from a Si Quantum Dot Hybrid Light-Emitting Diode. Appl. Phys. Lett. 2015, 106, 201102.

(2) Hirschman, K. D.; Tsybeskov, L.; Duttagupta, S. P.; Fauchet, P. M. Silicon-Based Visible Light-Emitting Devices Integrated into Microelectronic Circuits. Nature 1996, 384, 338-341.

(3) Govoni, M.; Marri, I.; Ossicini, S. Carrier Multiplication between Interacting Nanocrystals for Fostering Silicon-Based Photovoltaics. Nat. Photonics 2012, 6, 672-679.

(4) Cho, E.-C.; Park, S.; Hao, X.; Song, D.; Conibeer, G.; Park, S.-C.; Green, M. A. Silicon Quantum Dot/crystalline Silicon Solar Cells. Nanotechnology 2008, 19, 245201.

(5) Baxter, J. B.; Aydil, E. S. Nanowire-Based Dye-Sensitized Solar Cells. Appl. Phys. Lett. 2005, 86, 053114.

(6) Plass, R.; Pelet, S.; Krueger, J.; Gratzel, M.; Bach, U. Quantum Dot Sensitization of Organic-Inorganic Hybrid Solar Cells. I. Phys. Chem. B 2002, 106, 7578-7580.

(7) Gao, X. H.; Cui, Y. Y.; Levenson, R. M.; Chung, L. W. K.; Nie, S. M. In Vivo Cancer Targeting and Imaging with Semiconductor Quantum Dots. Nat. Biotechnol. 2004, 22, 969-976.

(8) Park, J.-H.; Gu, L.; von Maltzahn, G.; Ruoslahti, E.; Bhatia, S. N.; Sailor, M. J. Biodegradable Luminescent Porous Silicon Nanoparticles for in Vivo Applications. Nat. Mater. 2009, 8, 331-336.

(9) Buriak, J. M. Organometallic Chemistry on Silicon Surfaces: Formation of Functional Monolayers Bound through Si-C Bonds. Chem. Commun. 1999, 12, 1051-1060.
(10) Aldana, J.; Wang, Y. A.; Peng, X. G. Photochemical Instability of CdSe Nanocrystals Coated by Hydrophilic Thiols. I. Am. Chem. Soc. 2001, 123, 8844-8850.

(11) Trani, F.; Cantele, G.; Ninno, D.; Iadonisi, G. Tight-Binding Calculation of the Optical Absorption Cross Section of Spherical and Ellipsoidal Silicon Nanocrystals. Phys. Rev. B: Condens. Matter Mater. Phys. 2005, 72, 075423.

(12) Kocevski, V.; Eriksson, O.; Rusz, J. Transition between Direct and Indirect Band Gap in Silicon Nanocrystals. Phys. Rev. B: Condens. Matter Mater. Phvs. 2013, 87, 245401.

(13) Hapala, P.; K⿺̊sová, K.; Pelant, I.; Jelínek, P. Theoretical Analysis of Electronic Band Structure of 2- to 3-Nm Si Nanocrystals. Phys. Rev. B: Condens. Matter Mater. Phys. 2013, 87, 195420.

(14) Wilson, W. L.; Szajowski, P. F.; Brus, L. E. Quantum Confinement in Size-Selected, Surface Oxidized Silicon Nanocrystals. Science 1993, 262, 1242-1244.

(15) Pavesi, L.; Dal Negro, L.; Mazzoleni, C.; Franzo, G.; Priolo, F. Optical Gain in Silicon Nanocrystals. Nature 2000, 408, 440-444.

(16) Anthony, R.; Kortshagen, U. Photoluminescence Quantum Yields of Amorphous and Crystalline Silicon Nanoparticles. Phvs. Rev. B: Condens. Matter Mater. Phys. 2009, 80, 115407.

(17) Timmerman, D.; Valenta, J.; Dohnalova, K.; de Boer, W. D. A. M.; Gregorkiewicz, T. Step-like Enhancement of Luminescence Quantum Yield of Silicon Nanocrystals. Nat. Nanotechnol. 2011, 6, $710-713$.

(18) Beard, M. C.; Knutsen, K. P.; Yu, P.; Luther, J. M.; Song, Q.; Metzger, W. K.; Ellingson, R. J.; Nozik, A. J. Multiple Exciton Generation in Colloidal Silicon Nanocrystals. Nano Lett. 2007, 7, 2506-2512.

(19) Fischer, S. A.; Madrid, A. B.; Isborn, C. M.; Prezhdo, O. V. Multiple Exciton Generation in Small Si Clusters: A High-Level, $\underline{\mathrm{Ab}}$ Initio Studv. I. Phvs. Chem. Lett. 2010, 1, 232-237.

(20) Jaeger, H. M.; Hyeon-Deuk, K.; Prezhdo, O. V. Exciton Multiplication from First Principles. Acc. Chem. Res. 2013, 46, 12801289.

(21) Pi, X. D.; Mangolini, L.; Campbell, S. A.; Kortshagen, U. RoomTemperature Atmospheric Oxidation of Si Nanocrystals after HF Etching. Phvs. Rev. B: Condens. Matter Mater. Phvs. 2007, 75, 085423.

(22) Kanemitsu, Y.; Okamoto, S.; Otobe, M.; Oda, S. Photoluminescence Mechanism in Surface-Oxidized Silicon Nanocrystals. Phvs. Rev. B: Condens. Matter Mater. Phvs. 1997, 55, R7375.

(23) Wolkin, M. V.; Jorne, J.; Fauchet, P. M.; Allan, G.; Delerue, C. Electronic States and Luminescence in Porous Silicon Quantum Dots: The Role of Oxygen. Phvs. Rev. Lett. 1999, 82, 197-200.

(24) van Buuren, T.; Dinh, L. N.; Chase, L. L.; Siekhaus, W. J.; Terminello, L. J. Changes in the Electronic Properties of Si Nanocrystals as a Function of Particle Size. Phys. Rev. Lett. 1998, 80, 3803-3806.

(25) Hessel, C. M.; Henderson, E. J.; Kelly, J. A.; Cavell, R. G.; Sham, T.-K.; Veinot, J. G. C. Origin of Luminescence from Silicon Nanocrystals: A Near Edge X-ray Absorption Fine Structure (NEXAFS) and X-ray Excited Optical Luminescence (XEOL) Study of Oxide-Embedded and Free-Standing Systems. I. Phys. Chem. C 2008, 112, 14247-14254.

(26) Godefroo, S.; Hayne, M.; Jivanescu, M.; Stesmans, A.; Zacharias, M.; Lebedev, O. I.; Van Tendeloo, G.; Moshchalkov, V. V. Classification and Control of the Origin of Photoluminescence from Si Nanocrystals. Nat. Nanotechnol. 2008, 3, 174-178.

(27) de Boer, W. D. A. M.; Timmerman, D.; Dohnalova, K.; Yassievich, I. N.; Zhang, H.; Buma, W. J.; Gregorkiewicz, T. Red Spectral Shift and Enhanced Quantum Efficiency in Phonon-Free Photoluminescence from Silicon Nanocrystals. Nat. Nanotechnol. 2010, 5, 878-884.

(28) Liu, S.-M. Luminescent Silicon Nanoparticles Formed in Solution. I. Nanosci. Nanotechnol. 2008, 8, 1110-1125.

(29) Godefroo, S.; Hayne, M.; Jivanescu, M.; Stesmans, A.; Zacharias, M.; Lebedev, O. I.; Van Tendeloo, G.; Moshchalkov, V. V. Classification and Control of the Origin of Photoluminescence from Si Nanocrystals. Nat. Nanotechnol. 2008, 3, 174-178. 
(30) Dasog, M.; Yang, Z.; Regli, S.; Atkins, T. M.; Faramus, A.; Singh, M. P.; Muthuswamy, E.; Kauzlarich, S. M.; Tilley, R. D.; Veinot, J. G. C. Chemical Insight into the Origin of Red and Blue Photoluminescence Arising from Freestanding Silicon Nanocrystals. ACS Nano 2013, 7, 2676-2685.

(31) Puzder, A.; Williamson, A. J.; Grossman, J. C.; Galli, G. Surface Control of Optical Properties in Silicon Nanoclusters. L. Chem. Phys. 2002, 117, 6721-6729.

(32) Luppi, M.; Ossicini, S. Ab Initio Study on Oxidized Silicon Clusters and Silicon Nanocrystals Embedded in SiO2: Beyond the Quantum Confinement Effect. Phys. Rev. B: Condens. Matter Mater. Phys. 2005, 71, 035340.

(33) Kocevski, V.; Eriksson, O.; Rusz, J. Size Dependence of the Stability, Electronic Structure, and Optical Properties of Silicon Nanocrystals with Various Surface Impurities. Phvs. Rev. B: Condens. Matter Mater. Phys. 2015, 91, 125402.

(34) Ramos, L. E.; Furthmuller, J.; Bechstedt, F. Effect of Backbond Oxidation on Silicon Nanocrystallites. Phvs. Rev. B: Condens. Matter Mater. Phvs. 2004, 70, 033311.

(35) Vasiliev, I.; Chelikowsky, J. R.; Martin, R. M. Surface Oxidation Effects on the Optical Properties of Silicon Nanocrystals. Phvs. Rev. B: Condens. Matter Mater. Phys. 2002, 65, 121302.

(36) Puzder, A.; Williamson, A. J.; Grossman, J. C.; Galli, G. Computational Studies of the Optical Emission of Silicon Nanocrystals. I. Am. Chem. Soc. 2003, 125, 2786-2791.

(37) Pennycook, T. J.; Hadjisavvas, G.; Idrobo, J. C.; Kelires, P. C.; Pantelides, S. T. Optical Gaps of Free and Embedded Si Nanoclusters: Density Functional Theory Calculations. Phys. Rev. B: Condens. Matter Mater. Phvs. 2010, 82, 125310.

(38) Zhou, Z. Y.; Brus, L.; Friesner, R. Electronic Structure and Luminescence of 1.1-And 1.4-Nm Silicon Nanocrystals: Oxide Shell versus Hydrogen Passivation. Nano Lett. 2003, 3, 163-167.

(39) Chen, C. J. Introduction to Scanning Tunneling Microscopy, 2nd ed.; Oxford University Press: New York, 2008.

(40) Wolf, O.; Dasog, M.; Yang, Z.; Balberg, I.; Veinot, J. G. C.; Millo, O. Doping and Quantum Confinement Effects in Single $\mathrm{Si}$ Nanocrystals Observed by Scanning Tunneling Spectroscopy. Nano Lett. 2013, 13, 2516-2521.

(41) Kislitsyn, D. A.; Gervasi, C. F.; Allen, T.; Palomaki, P. K. B.; Hackley, J. D.; Maruyama, R.; Nazin, G. V. Spatial Mapping of SubBandgap States Induced by Local Nonstoichiometry in Individual Lead Sulfide Nanocrystals. L. Phvs. Chem. Lett. 2014, 5, 3701-3707.

(42) Gervasi, C. F.; Kislitsyn, D. A.; Allen, T. L.; Hackley, J. D.; Maruyama, R.; Nazin, G. V. Diversity of Sub-Bandgap States in LeadSulfide Nanocrystals: Real-Space Spectroscopy and Mapping at the Atomic-Scale. Nanoscale 2015, 7, 19732-19742.

(43) Hackley, J. D.; Kislitsyn, D. A.; Beaman, D. K.; Ulrich, S.; Nazin, G. V. High-Stability Cryogenic Scanning Tunneling Microscope Based on a Closed-Cycle Cryostat. Rev. Sci. Instrum. 2014, 85, 103704.

(44) Hadjisavvas, G.; Remediakis, I. N.; Kelires, P. C. Shape and Faceting of $\mathrm{Si}$ Nanocrystals Embedded in a- $\mathrm{SiO}_{2}$ : A Monte Carlo Study. Phys. Rev. B: Condens. Matter Mater. Phvs. 2006, 74, 165419.

(45) Wang, Y. Q.; Smirani, R.; Ross, G. G. Nanotwinning in Silicon Nanocrystals Produced by Ion Implantation. Nano Lett. 2004, 4, 2041-2045.

(46) Hapala, P.; Kusova, K.; Pelant, I.; Jelinek, P. Theoretical Analysis of Electronic Band Structure of 2- to 3-Nm Si Nanocrystals. Phys. Rev. B: Condens. Matter Mater. Phys. 2013, 87, 195420.

(47) Sun, Z.; Swart, I.; Delerue, C.; Vanmaekelbergh, D.; Liljeroth, P. Orbital and Charge-Resolved Polaron States in CdSe Dots and Rods Probed by Scanning Tunneling Spectroscopy. Phvs. Rev. Lett. 2009, 102, 196401.

(48) Chiu, S.-K.; Manhat, B. A.; DeBenedetti, W. J. I.; Brown, A. L.; Fichter, K.; Vu, T.; Eastman, M.; Jiao, J.; Goforth, A. M. Aqueous RedEmitting Silicon Nanoparticles for Cellular Imaging: Consequences of Protecting against Surface Passivation by Hydroxide and Water for Stable Red Emission. I. Mater. Res. 2013, 28, 216-230.

(49) Hessel, C. M.; Henderson, E. J.; Veinot, J. G. C. Hydrogen Silsesquioxane: A Molecular Precursor for Nanocrystalline $\mathrm{Si}^{-S_{\mathrm{O}}}{ }_{2}$
Composites and Freestanding Hydride-Surface-Terminated Silicon Nanoparticles. Chem. Mater. 2006, 18, 6139-6146.

(50) Weeks, S. L.; Chaukulkar, R. P.; Stradins, P.; Agarwal, S. Photoluminescence Behavior of Plasma Synthesized Si Nanocrystals Oxidized at Low Temperature in Pure $\mathrm{O}_{2}$ and $\mathrm{H}_{2} \mathrm{O}$. I. Vac. Sci. Technol., A 2014, 32, 050604.

(51) Brawand, N. P.; Voros, M.; Galli, G. Dangling Bonds are a Cause of B-Type Blinking in Si Nanoparticles. Nanoscale 2015, 7, 3737-3744

(52) Miller, J. B.; Dandu, N.; Velizhanin, K. A.; Anthony, R. J.; Kortshagen, U. R.; Kroll, D. M.; Kilina, S.; Hobbie, E. K. Enhanced Luminescent Stability through Particle Interactions in Silicon Nanocrystal Aggregates. ACS Nano 2015, 9, 9772-9782.

(53) Ordejon, P.; Artacho, E.; Soler, J. M. Self-Consistent Order-N Density-Functional Calculations For Very Large Systems. Phvs. Rev. B: Condens. Matter Mater. Phvs. 1996, 53, R10441.

(54) Soler, J. M.; Artacho, E.; Gale, J. D.; Garcia, A.; Junquera, J.; Ordejon, P.; Sanchez-Portal, D. The SIESTA Method for Ab initio Order-N Materials Simulation. I. Phvs.: Condens. Matter 2002, 14, 2745-2779. 